\title{
Osteopontin is a tumor autoantigen in prostate cancer patients
}

\author{
TATIANA M. TILLI ${ }^{1}$, ELOÍSIO A. SILVA ${ }^{2}$, LÍVIA C. MATOS ${ }^{3}$, DOUGLAS V. FAGET ${ }^{1}$, \\ BIANCA F.P. DIAS ${ }^{1}$, JULIANA S.P. VASCONCELOS ${ }^{2}$, YASUYUKI YOKOSAKI ${ }^{5}$ and ETEL R.P. GIMBA ${ }^{1,4}$ \\ ${ }^{1}$ Programa de Medicina Experimental and Biologia Celular and Post Graduation Program in Oncology, \\ Coordenação de Pesquisa, Instituto Nacional de Câncer; ${ }^{2}$ Serviço de Urologia, Hospital Universitário Pedro Ernesto, \\ Universidade do Estado do Rio de Janeiro, Rio de Janeiro; ${ }^{3}$ Universidade de Brasília, Brasília-DF; \\ ${ }^{4}$ Universidade Federal Fluminense, Polo Universitário de Rio das Ostras, Rio das Ostras, RJ, Brazil; \\ ${ }^{5}$ Cell-Matrix Frontier Laboratory Biomedical Research Building, Hiroshima University, Minamiku, Hiroshima, Japan
}

Received July 21, 2010; Accepted October 4, 2010

DOI: $10.3892 / \mathrm{ol} .2010 .211$

\begin{abstract}
Anti-tumor antibodies act as biomarkers for the early diagnosis of prostate cancer (PCa). Osteopontin (OPN) is overexpressed in PCa cells and contributes to the progression of the disease. This study aimed to evaluate whether OPN evokes a humoral immune response in PCa patients and whether the reactivity levels of anti-OPN antibodies may be used to better differentiate $\mathrm{PCa}$ from benign and healthy donor plasma samples. Plasma samples from biopsy-proven $\mathrm{PCa}$ patients (29), benign prostate hyperplasia (BPH) (18) and control healthy donors (HD) (30) were tested by immunoblots using the recombinant human OPN. The frequency of anti-OPN antibodies was significantly higher in $\mathrm{PCa}(66 \%)$ plasma samples as compared to BPH (33\%) and HD controls (10\%). Anti-OPN antibodies were detected in a high proportion of plasma samples from patients with a Gleason score of less than 6 $(57 \%)$, prostate-specific antigen levels lower than $10 \mathrm{ng} / \mathrm{ml}$ (67\%) and pT2 organ-confined disease (70\%), suggesting that anti-OPN antibodies may be used as an early serum marker for PCa. To the best of our knowledge, this is the first description of OPN as a tumor autoantigen and one of the most reactive individual autoantigens described thus far. These data support the inclusion of OPN in a multiplex of tumor antigens in order to perform antibody profiling in $\mathrm{PCa}$ as well as in other malignancies overexpressing OPN.
\end{abstract}

\section{Introduction}

Early and specific diagnosis is crucial for the successful treatment of prostate cancer (PCa). Novel biomarkers are required

Correspondence to: Dr Etel R.P. Gimba, Divisão de Medicina Experimental, Coordenação de Pesquisa, Instituto Nacional de Câncer/ UFF-Polo Universitário de Rio das Ostras, Rua André Cavalcante, 37-sexto andar, CEP 20231-050, Rio de Janeiro-RJ, Brazil

E-mail: egimba@inca.gov.br; etelgimba@hotmail.com

Key words: osteopontin, tumor antigen, autoantibodies, prostate cancer to complement prostate-specific antigen (PSA) measurements by enhancing its diagnostic and prognostic performance. It is crucial to define additional clinical markers for the accurate detection of $\mathrm{PCa}$ and differential diagnosis of benign prostatic hyperplasia (BPH) $(1,2)$.

One of the strategies for cancer serum biomarker development has been the detection of autoantibodies produced against tumor antigens in patient sera $(2,3)$. This approach measures the autoantibodies for a spectrum of tumor antigens, with sensitivity and specificity exceeding those obtained using the conventional circulating tumor antigen (4). A number of asymptomatic individuals who develop cancer exhibit circulating autoantibodies preceding clinical onset of the disease, indicating that immune response against tumor antigens is an early event in oncogenesis (5). The expression of tumorassociated antigens is the result of various events, including mutation, overexpression or altered expression patterns. In addition, autoantibody responses in cancer were correlated with patient survival and patient response to treatment (6-8). The identification of immunologically identified proteins that are specific or associated with PCa may lead to the development of specific targets for diagnosis, provide novel insights into the biology of prostate carcinogenesis and describe novel potential targets for immunotherapy-based vaccines.

Osteopontin (OPN) is a glycoprotein involved in a range of physiological and pathological processes. Extensive research has elucidated the pivotal role of OPN in regulating the cell signaling that controls tumor progression and metastasis (9-11). Circulating OPN has been positively associated with $\mathrm{PCa}$ bone metastases and negatively correlated with survival and other tumor burden characteristics (11-14). Overexpression of OPN in PCa cell lines induced increased proliferation, invasion and, most notably, enhanced the ability of these cells to intravasate blood vessels (15).

It has been shown that OPN expression is already deregulated in early neoplastic lesions (15). However, to the best of our knowledge, the evaluation of circulating OPN levels in the early stages of PCa and its potential use as a sensitive marker for PCa diagnosis has yet to be described. In order to evaluate the early expression of OPN in tumor conditions, anti-OPN antibodies in the early stages of tumor 
development should be detected. Anti-OPN antibodies in autoimmune diseases were previously described (16), suggesting that OPN is immunogenic and that loss of immune tolerance occurs in such pathological conditions. Based on these data, it was hypothesized that in early tumor development, circulating OPN triggers humoral immune responses. Using anti-OPN antibody immune responses as biomarkers may be noteworthy, since they represent a form of biological amplification of signals that are otherwise weak, especially in the early stages of various types of cancer.

The present study investigated whether OPN initiates an immune response in PCa patients by evaluating the presence of anti-OPN antibodies in plasma samples from $\mathrm{PCa}$ patients. We then compared the frequency of reactivity of anti-OPN antibodies between PCa, BPH and HD plasma samples. Furthermore, certain properties of anti-OPN antibodies as potential serum markers for PCa were evaluated.

\section{Materials and methods}

Patients. Plasma from biopsy-proven clinically-localized PCa patients was collected prior to prostatectomy and stored in aliquots at $-80^{\circ} \mathrm{C}$. Plasma samples were obtained from 29 biopsy-proven PCa patients (52-79 years of age, median 67), 18 patients with benign prostate hyperplasia (BPH) (62-87 years of age, median 72) and 30 healthy male donors (HD) (18-36 years of age, median 25) with no history of cancer or any other prostatic or auto-immune diseases. The HD samples were collected from non-hospitalized volunteers in Rio de Janeiro and used as control samples. The BPH cases corresponded to patients that were submitted to surgical treatment by open adenomectomy. The local Ethics Committee approved this study and the participants provided informed consent. Table I summarizes the data from the PCa patients included in the study.

Recombinant osteopontin. A GST-OPN fusion construct containing the complete OPN cDNA in the plasmid pGEX-6P2 (17) was used to generate the recombinant OPN (rOPN-GST), as previously described $(17,18)$. GST-OPN and GST recombinant proteins were purified under non-denaturing conditions using an in batch GST Purification Module (Amersham Biosciences, Piscataway, NJ, USA).

Western blotting for anti-osteopontin antibodies. Plasma IgGs against bacterial proteins were adsorbed by incubating bacterial extracts and plasma samples at a proportion of 1:1 $\mathrm{vol} / \mathrm{vol}$ for $2 \mathrm{~h}$ at room temperature with gentle agitation. For immunoblot assays, $50 \mu \mathrm{g}$ of GST-OPN and GST-purified recombinant proteins were subjected to electrophoresis via a $12 \%$ SDS-polyacrylamide gel and then transferred onto nitrocellulose membranes using the mini-PROTEAN 3 (Bio-Rad) system. Comparative immunoblot assays were performed simultaneously, using the same OPN recombinant protein batch. The membranes were probed with plasma samples diluted 1:200 in 1X PBS containing 0.05\% Tween-20 containing 5\% non-fat dry milk (PBSTNF) and subsequently with anti-human $\mathrm{IgG}$ alkaline phosphatase conjugate (Sigma, St. Louis, MO, USA) diluted 1:30,000 in PBSTNF. Immunoreactive bands were then visualized by the addition
Table I. Clinical and pathological data of the PCa patients included in this study and their anti-OPN reactivity levels.

\begin{tabular}{lcc}
\hline Variable & $\begin{array}{c}\text { No. of } \\
\text { patients }(\%)\end{array}$ & $\begin{array}{c}\text { Anti-OPN antibody } \\
\text { reactivity }(\%)\end{array}$ \\
\hline Total cases of PCa & 29 & $18 / 29(62)$ \\
Gleason score & $14(48)$ & $8 / 14(57)$ \\
$\leq 6$ & $5(17)$ & $4 / 5(80)$ \\
$7(3+4)$ & $4(14)$ & $4 / 5(80)$ \\
$7(4+3)$ & $6(21)$ & $3 / 6(50)$ \\
$\geq 8$ & & \\
PSA level before surgery & & $10 / 15(67)$ \\
Mean (median, range) & $15(68)$ & $6 / 7(86)$ \\
$<10$ ng/ml & $7(32)$ & $7 / 10(70)$ \\
$\geq 10$ ng/ml & & $8 / 12(67)$ \\
Pathology pT stage & $10(45)$ & $3 / 3(100)$ \\
pT2a - pT2b - pT2c & $12(55)$ & $0 / 19(0)$ \\
pT3a - pT3b & & \\
Lymph node status ${ }^{\mathrm{a}}$ & $3(14)$ & \\
Positive & $19(86)$ & \\
Negative & &
\end{tabular}

aData were available for 22 patients only; ${ }^{b}$ Data were available for 27 patients only. PSA, prostate specific antigen.

of nitroblue tetrazolium and 5-bromo-4-chloro-3-indolylphosphate (NBT/BCIP; Sigma) substrates.

Assays with OPN monoclonal antibody were performed in identical conditions to those described above, using the OPN $\mathrm{mAb}$ (Chemicon, Canada, USA) in a 1:2,500 dilution followed by incubation in a 1:5,000 dilution of anti-rat $\operatorname{IgG}$ conjugated to horseradish peroxidase (HRP). Immunoreactive complexes were developed with the ECL Plus System (Amersham Biosciences) after exposure to Kodak BioMax Light Film.

Enzyme linked immunosorbent assay (ELISA). Recombinant OPN fusion protein solution $(50 \mu \mathrm{l})$ at a concentration of $25 \mu \mathrm{g} / \mathrm{ml}$ in coating buffer carbonate/bicarbonate $0.1 \mathrm{M}$ $\mathrm{pH} 9.6$ was added to 96 -well plates and incubated overnight at $40^{\circ} \mathrm{C}$. The plasma samples were serially diluted from 1:100 to $1: 1,000$ in PBSTNF. The plates were incubated for $1 \mathrm{~h}$ at room temperature on an orbital agitator and then washed with 1X PBS. HRP-conjugated human IgG-specific secondary antibody (1:4,000 in PBSTNF) was added and the plates were incubated for one more hour at room temperature. After being washed five times in $1 \mathrm{X}$ PBS, 3,3', 5,5'-tetramethyl-benzidine (TMB; Invitrogen) was added. The reactions were stopped by the addition of $100 \mu \mathrm{l}$ of $0.5 \mathrm{~N} \mathrm{H}_{2} \mathrm{SO}_{4}$ per well. Absorbance values were read at $450 \mathrm{~nm}$ using a microplate reader SpectraMax 90 (Molecular Devices, Sunnyvale, CA, USA).

Sensitivity, specificity and predictive values. In order to evaluate the potential use of anti-OPN antibodies as serum markers for $\mathrm{PCa}$, the sensitivity, specificity, positive predictive 


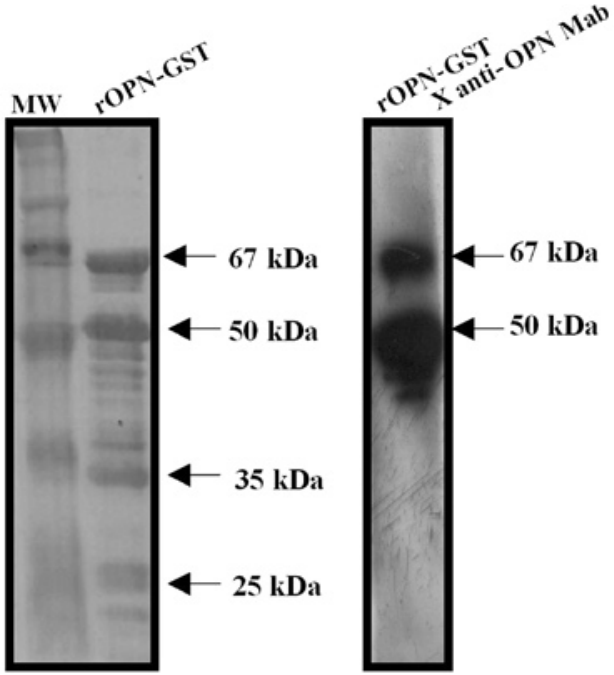

Figure 1. Expression and purification of rOPN-GST fusion protein. rOPN was produced as a fusion protein with GST in E. coli BL21. (Left panel) Purified rOPN-GST was separated by $12 \%$ SDS-PAGE and stained with Coomassie brillant blue. (Right panel) The separated fusion proteins were transferred onto nitrocellulose membranes and then incubated with an anti-OPN monoclonal antibody.

value (PPV) and negative predictive value (NPV) were calculated, as previously described (12).

Statistical analysis. Statistical analysis was performed with SPSS 14.0 and Microsoft Excel. The Chi-square test was used for the statistically significant differences in the humoral immune response to OPN among patients with $\mathrm{PCa}, \mathrm{BPH}$ and the $\mathrm{HD}$ control groups. The associations between the humoral immune response against OPN and the various clinical and pathological characteristics were determined using the Spearman's correlation test. $\mathrm{P} \leq 0.05$ was considered to be statistically significant.

\section{Results}

In order to evaluate the presence of autoantibodies against OPN in PCa plasma samples, a recombinant human osteopontin (rOPN) was used as an antigen in immunoblot assays. Purified rOPN-GST presented the predicted molecular weight of $67 \mathrm{kDa}$ as a result of GST (28 kDa) plus the full-length human OPN protein (39 kDa) (Fig. 1, left panel). Additional protein fragments were observed in SDS-PAGE, likely resulting from the degradation of rOPN-GST during antigen preparation. Hence, purified rOPN-GST presented four major bands. The upper band corresponds to the full-length OPN $(67 \mathrm{kDa})$ and the three additional lower protein fragments $(50$, 35 and $25 \mathrm{kDa}$ ) were evidently degradation products. The 67 and $50 \mathrm{kDa}$ major protein bands were reactive to an anti-OPN monoclonal antibody, confirming OPN identity (Fig. 1, right panel). Conversely, the remaining protein fragments were not reactive, suggesting that a loss of the OPN epitope identified by this monoclonal antibody occurred via the lower degradation products.

Using the rOPN-GST described above, immunoblot assays were performed to evaluate autoantibody responses to rOPN in plasma samples from $29 \mathrm{PCa}$ patients with different clinical and pathological staging, 18 from patients with $\mathrm{BPH}$ and 30 from HD controls. Fig. 2 shows representative immunoblot assays of these tested plasma samples.
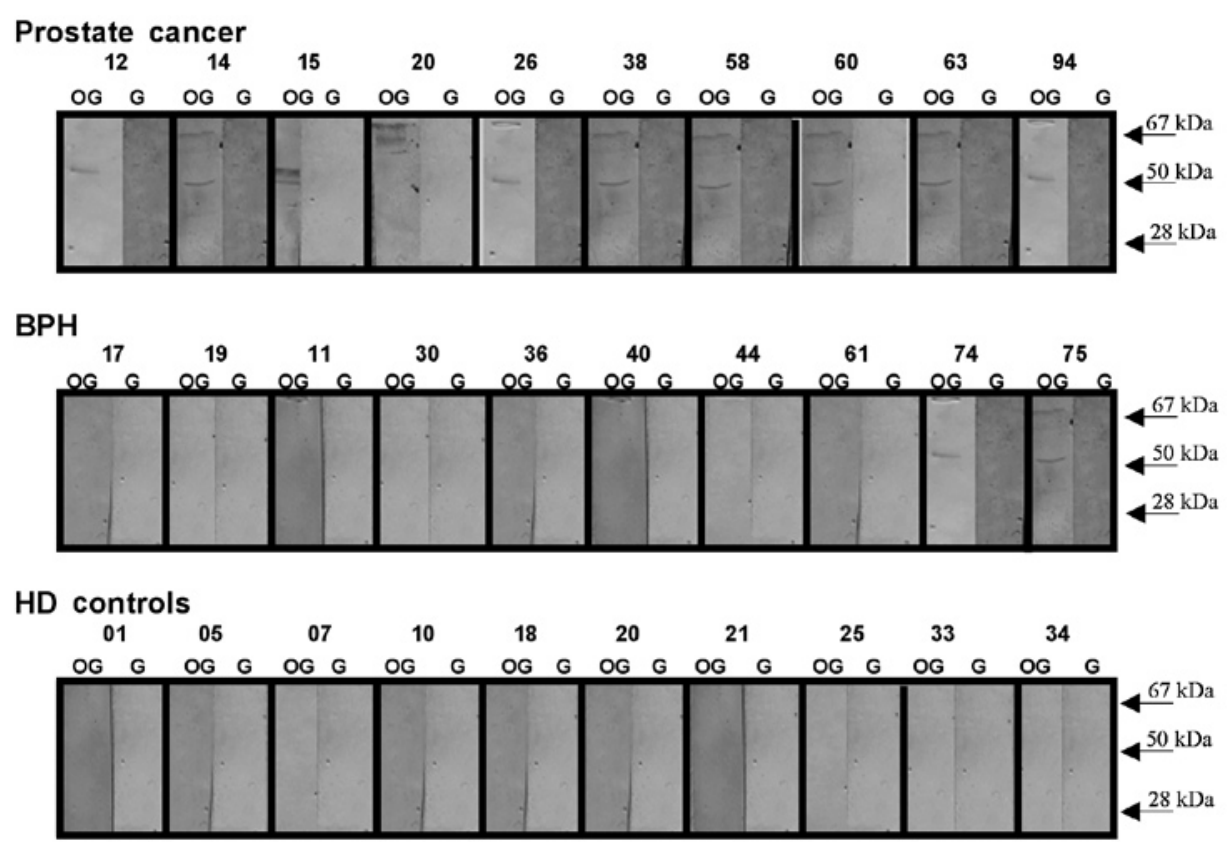

Figure 2. PCa patients present higher reactivity levels of anti-OPN antibodies as compared to BPH and the HD controls. A representative immunoblot of plasma samples assayed for their reactivity to recombinant OPN is shown. Plasma samples from 29 biopsy-proven PCa, 18 BPH and 30 HD were tested. The plasma samples were tested in 1:200 dilution and the antigen-antibody complexes were probed with AP-conjugated anti-human IgG antibody. The assays were performed simultaneously using the same batch of recombinant OPN. The representative plasma samples tested are numbered above each panel. The detection of immunoreactive bands was performed using NBT/BCIP as AP substrates. The two major reactive rOPN-GST (lane OG) protein fragments and GST alone (lane G) were used as a negative control and their molecular weight sizes are indicated by the arrows. None of the tested pre-adsorbed plasma samples were reactive for GST. 
Table II. Distribution of positive and negative reactive immunoblot results for anti-OPN antibodies between PCa and non-PCa control groups (BPH and HD).

\begin{tabular}{lccr}
\hline Immunoblot results for anti-OPN antibodies & PCa $(\mathrm{n}=29)(\%)$ & $\mathrm{BPH}(\mathrm{n}=18)(\%)$ & HD controls $(\mathrm{n}=30)(\%)$ \\
\hline Positive & $19 / 29(62)^{\mathrm{a}}$ & $6 / 19(32)^{\mathrm{b}}$ & $3 / 30(10)$ \\
Negative & $10 / 29(38)$ & $13 / 19(68)$ & $27 / 30(90)$ \\
Total & 29 & 18 & 30 \\
\hline
\end{tabular}

${ }^{\text {aP }}\left(\chi^{2} \geq 3.67\right)=0.0554$ compared to BPH and $\mathrm{P}\left(\chi^{2} \geq 17.59\right)=0.00003$ compared to HD controls. ${ }^{\text {b }} \mathrm{P}\left(\chi^{2} \geq 17.59\right)=0.00015$ compared to HD controls. $\mathrm{BPH}$, benign prostate hyperplasia; $\mathrm{HD}$, healthy male donors; $\mathrm{PCa}$, prostate cancer.

Table III. Association between OPN humoral response and clinical and histopathological characteristics ${ }^{\mathrm{a}}$.

\begin{tabular}{lcccc}
\hline Variable & Analysis & PSA & Gleason score & Pathological stage \\
\hline $\begin{array}{l}\text { OPN humoral } \\
\text { response }\end{array}$ & $\begin{array}{c}\text { Spearman's correlation } \\
\text { test (P-value) }\end{array}$ & 0.279 & 0.719 & 0.805 \\
\hline
\end{tabular}

aHumoral response was assessed by immunoblot assays. PSA, prostate-specific antigen; OPN, osteopontin.

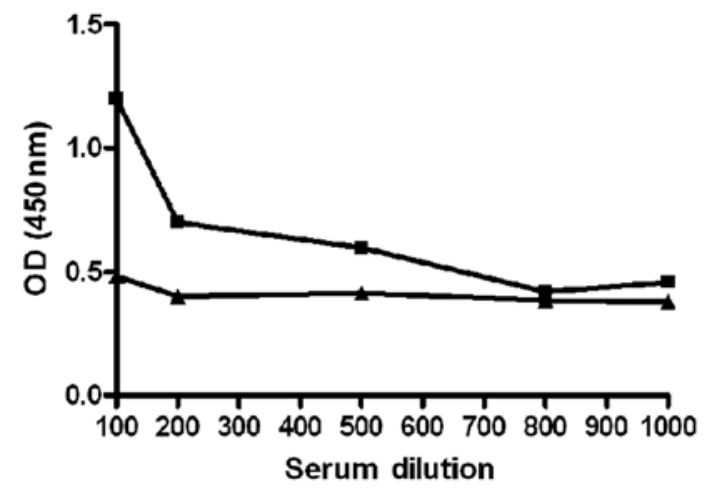

Figure 3. Anti-OPN antibodies are specific for human OPN. Titration curves were performed using an ELISA assay with an anti-OPN reactive prostate cancer plasma sample ( $\mathbf{\square})$ and a healthy control plasma sample ( $\mathbf{\Delta})$ tested against the recombinant human OPN. The samples were classified as positive and negative for anti-OPN antibodies, respectively, in the Western blot analysis. Plasma samples were serially diluted from 1:100 to 1:1,000 in PBSTNF, as described in Materials and methods.

Antibodies against rOPN-GST were detected in 19/29 (66\%), 6/18 (33\%) and 3/30 (10\%) plasma samples from PCa, $\mathrm{BPH}$ and HD, respectively (Table II). The immunoreactivity to rOPN-GST was specific for the OPN portion, since no immunoreactivity to GST was observed using pre-adsorbed plasma samples (Materials and methods) from patients with $\mathrm{PCa}, \mathrm{BPH}$ or from the HD controls (Fig. 2, right panels of each plasma sample tested). In order to validate the specificity of the humoral immune response to OPN observed in the immunoblot tests, ELISA titration curves of anti-OPN reactive and non-reactive plasma samples (Fig. 3) were performed. Imunoblot-positive PCa plasma samples exhibited a decrease of anti-OPN reactivity according to successive plasma dilution. The same was not observed for the HD plasma sample, which exhibited negative immunoblot results. These results confirm the specificity of the humoral immune response against OPN detected by these immunoblot assays.

The majority of the plasma samples were reactive to both the 67- and 50-kDa rOPN-GST products. However, certain samples were reactive for only one of the major protein molecular weights (e.g., PCa plasma samples nos. 12 and 20 in Fig. 2, which were reactive for only the $50-$ and $67-\mathrm{kDa}$ rOPN-GST molecular weights, respectively). This differential reactivity may be the result of an individual humoral immune response to different osteopontin antigenic domains. Notably, anti-OPN reactive plasma samples were significantly more frequent in $\mathrm{PCa}(66 \%)$, as compared to $\mathrm{BPH}$ patients $(33 \%)$ and the HD (10\%) control group (Table II).

Certain diagnostic properties of anti-OPN antibodies were then evaluated as potential serum markers to detect $\mathrm{PCa}$ and to discriminate PCa from $\mathrm{BPH}$ and HD controls. The results from the screening were used to calculate sensitivity, specificity and predictive values. These characteristics were based on the premise that control patients (true negative group) are those with BPH or the HD controls. Our analysis resulted in a sensitivity of $66 \%$, specificity of $81 \%$, PPV of $68 \%$ (positive test) and NPV of $80 \%$ (negative test).

To evaluate whether the presence of anti-OPN antibodies is an early event in $\mathrm{PCa}$ progression, anti-OPN reactivity levels were investigated based on certain clinical data from PCa patients whose plasma samples were tested (Table I). Of the patients presenting a pT2 stage ( $45 \%$ of the samples), $70 \%$ were positive for anti-OPN antibodies. A total of $57 \%$ of patients presenting tumors with a Gleason score of less than 6 were positive for anti-OPN antibodies. Additionally, the data showed that $67 \%$ of patients included in this study, with a pre-surgery PSA value of less than $10 \mathrm{ng} / \mathrm{ml}$, were also positive for anti-OPN antibodies. The results showed that even PCa patients with organ-confined disease (pT2 pathological 
staging), PSA plasma levels lower than $10 \mathrm{ng} / \mathrm{ml}$ and with a Gleason score of less than 6, exhibited humoral immune responses against OPN.

The association between the presence of anti-OPN antibodies and clinical and pathological characteristics, including PSA level, Gleason score and TNM staging, was evaluated. No statistically significant association was found between humoral immune response against OPN or any of these characteristics (Table III).

\section{Discussion}

The identification and molecular characterization of selfantigens expressed by human malignancies are an active field in tumor immunology. The approach employed to measure autoantibodies for a spectrum of tumor antigens results in sensitivity and specificity results exceeding those obtained using the measurement of tumor antigen levels $(19,20)$. A number of asymptomatic individuals who develop cancer exhibit circulating autoantibodies preceding the clinical onset of the disease, suggesting that an immune response against tumor antigens is an early event in oncogenesis (5). In addition, autoantibody responses in cancer have been correlated with patient survival and response to treatment (6-8).

Data indicate OPN to be a significant immunogen in various pathological conditions. Dysregulated OPN expression in certain autoimmune diseases is possibly correlated to the break of immune tolerance to this self protein. Anti-OPN antibodies are produced in such pathological conditions, but OPN is a weak immunogen $(0.95-15 \%)(16,21)$. Healthy individuals presented very low or absent anti-OPN reactivity levels as compared to autoimmune diseases $(16,22)$. Similar mechanisms mediate tolerance to tumor antigens and self-antigens in order to avoid autoimmunity, and numerous autoimmune phenomena were reported in malignancies $(23,24)$. Based on this assumption, we evaluated whether the overexpression of OPN in tumor conditions was able to break immune tolerance against this protein, as was observed in certain autoimmune diseases, thereby triggering humoral immunity in cancer patients. Additionally, using PCa serum samples as models to achieve this objective, we investigated whether anti-OPN antibodies were potential serum markers for this disease. The search for autoantibodies against tumor antigens as serological markers for cancer diagnosis has been a long-term undertaking of PCa investigators $(2,25)$. A number of overexpressed antigens were reported to initiate spontaneous humoral and cellular immune responses in PCa (25). We proposed that OPN may be one such example, in that occasionally it is one of the antigens triggering humoral immune response in PCa patients.

The present study showed that the frequency of patients presenting autoantibody to OPN was significantly higher in PCa (66\%) plasma samples than in BPH (33\%) and the HD (10\%) control group. BPH is a frequent cause of lower urinary symptoms, with a prevalence of $50 \%$ by the sixth decade of life. It is anticipated that in the next few years the male population aged 65 years and older is due to increase, and it may become crucial to distinguish male individuals with $\mathrm{BPH}$ from those with PCa warranting clinical intervention. To achieve this aim, a definition of the additional clinical markers for accurate detection of PCa and differential diagnosis of $\mathrm{BPH}$ is required $(1,2)$. According to our data, anti-OPN antibodies may be appropriate serum markers that, together with other markers, may aid to better distinguish PCa from BPH and $\mathrm{HD}$ controls. BPH patients presenting anti-OPN antibodies should be followed up in order to evaluate whether or not they present a non-diagnosed PCa. The observed difference in frequency of anti-OPN antibodies in the three groups of individuals evaluated in this study may be the result of a higher OPN expression level in PCa in comparison to BPH and normal prostate tissues, as previously described $(15,26)$.

To the best of our knowledge, no previously published study exists regarding the direct identification of autoantibodies against OPN in PCa or other types of cancer. Thus, our data showed for the first time that OPN triggers antibody immunity in cancer and that OPN is one of the most reactive individual PCa tumor-associated antigens described thus far, presenting a prevalence of anti-OPN antibody frequency of $66 \%$ among PCa plasma samples. Currently, the most frequent anti-tumor antibodies against single antigens in $\mathrm{PCa}$ are for $\alpha$-methyl-acyl coA racemase (AMACR) (70\%), huntingtin (46\%), p90 (30.8\%) and FLJ23438 (37\%) (27-30). In combination with these higher reactive antigens and others yet to be described, antigen panels or arrays may be optimized, including OPN, in order to obtain high sensitivity and specificity levels, as previously described $(19,20,29,30)$. Additionally, we observed that the frequency of PCa patients presenting anti-OPN antibodies (66\%) is higher than that previously observed for patients presenting with autoimmune conditions $(0.95-15 \%)(16,21)$. It is likely that this phenomenon is related to the higher level of OPN expression in tumors as compared to autoimmune conditions.

The evaluation of anti-OPN antibodies as potential serum markers for PCa resulted in a sensitivity of $66 \%$, specificity of $81 \%$, PPV of $68 \%$ and NPV of $80 \%$, values considered reasonable for a humoral immune response against an individual antigen. Compared to the measurement of total PSA properties as a serum marker, which has been described in the range of $55-75 \%$ for specificity and $70-95 \%$ for sensitivity (31), anti-OPN antibodies may complement these properties to better define PCa diagnosis. Our data provides similar sensitivity and specificity results and higher PPV and NPV values when compared to circulating OPN as a marker for PCa (12). However, as opposed to our data, this study (12) focused on plasma samples from metastatic patients. Patients presenting early developing tumors have not been included, thereby hampering appropriate comparisons.

Our findings support the potential use of anti-OPN antibodies as additional serum markers to better aid in the early detection of PCa. The fact that anti-OPN antibodies were also detected in a high proportion of PCa patients presenting a low Gleason score, PSA values less than $10 \mathrm{ng} / \mathrm{ml}$ and PCa-confined disease, suggests that an early anti-OPN immune response occurs in PCa development.

A correlation between the humoral immune response to OPN and clinical and pathological characteristics, such as PSA level, Gleason score and TNM staging, was not found in this study, similar to results reported for other tumor antigens, including FLJ23438 and AMACR $(28,30)$. This suggests that the humoral immune response to certain tumor antigens is an independent factor for the evaluation of PCa progression. 
In conclusion, this study describes for the first time that OPN triggers humoral immunity in cancer. We provide evidence that anti-OPN antibodies are potential serum markers for early $\mathrm{PCa}$ stage and for the differential diagnosis of $\mathrm{PCa}$ and BPH. As a strong immunogenic autoantigen in PCa, OPN potentially initiates autoantibodies in other malignancies overexpressing this protein, justifying further investigation in other systems. OPN, in association with other tumor proteins, may be applied to antigen panels in order to achieve more favorable sensitivity and specificity results in the diagnosis of $\mathrm{PCa}$.

\section{Acknowledgements}

This study was supported by the Swiss Bridge Foundation, PRONEX-Rio, FAPERJ, CNPq, CAPES, FAF and INCa-MS.

\section{References}

1. Miller $\mathrm{J}$ and Tarter TH: Update on the use of dutasteride in the management of benign prostatic hypertrophy. Clin Interv Aging 2: 99-104, 2007.

2. Taylor BS, Pal M, Yu J, et al: Humoral response profiling reveals pathways to prostate cancer progression. Mol Cell Proteomics 7: 600-611, 2008.

3. Lu H, Goodell V and Disis ML: Humoral immunity directed against tumor-associated antigens as potential biomarkers for the early diagnosis of cancer. J Proteome Res 7: 1388-1394, 2008.

4. Cho-Chung YS: Autoantibody biomarkers in the detection of cancer. Biochim Biophys Acta 1762: 587-591, 2006.

5. Lubin R, Schlichtholz B, Teillaud JL, et al: p53 antibodies in patients with various types of cancer: assay, identification and characterization. Clin Cancer Res 1: 1463-1469, 1995.

6. Storr SJ, Chakrabarti J, Barnes A, et al: Use of autoantibodies in breast cancer screening and diagnosis. Expert Rev Anticancer Ther 6: 1215-1223, 2006.

7. Bachelot T, Ratel D, Menetrier-Caux C, et al: Autoantibodies to endostatin in patients with breast cancer: correlation to endostatin levels and clinical outcome. Br J Cancer 94: 1066-1070, 2006.

8. Vural B, Chen LC, Saip P, et al: Frequency of SOX Group B (SOX1, 2, 3) and ZIC2 antibodies in Turkish patients with small cell lung carcinoma and their correlation with clinical parameters. Cancer 103: 2575-2583, 2005.

9. Jacobs JP, Pettit AR, Shinohara ML, et al: Lack of requirement of osteopontin for inflammation, bone erosion, and cartilage damage in the $\mathrm{K} / \mathrm{BxN}$ model of autoantibody-mediated arthritis. Arthritis Rheum 50: 2685-2694, 2004.

10. Stromnes IM and Goverman JM: Osteopontin-induced survival of T cells. Nat Immunol 8: 19-20, 2007.

11. Shevde LA, Das S, Clark DW, et al: Osteopontin: an effector and an effect of tumor metastasis. Curr Mol Med 10: 71-81, 2010.

12. Fedarko NS, Jain A, Karadag A, et al: Elevated serum bone sialoprotein and osteopontin in colon, breast, prostate, and lung cancer. Clin Cancer Res 7: 4060-4066, 2001
13. Hotte SJ, Winquist EW, Stitt L, et al: Plasma osteopontin: associations with survival and metastasis to bone in men with hormone-refractory prostate carcinoma. Cancer 95: 506-512, 2002.

14. Wai PY and Kuo PC: Osteopontin: regulation in tumor metastasis. Cancer Metastasis Rev 27: 103-118, 2008.

15. Forootan SS, Foster CS, Aachi VR, et al: Prognostic significance of osteopontin expression in human prostate cancer. Int $\mathrm{J}$ Cancer 118: 2255-2261, 2006

16. Sakata M, Tsuruha JI, Masuko-Hongo K, et al: Autoantibodies to osteopontin in patients with osteoarthritis and rheumatoid arthritis. J Rheumatol 28: 1492-1495, 2001.

17. Yokosaki Y, Matsuura N, Sasaki T, et al: The integrin alpha(9) beta(1) binds to a novel recognition sequence (SVVYGLR) in the thrombin-cleaved amino-terminal fragment of osteopontin. J Biol Chem 274: 36328-36334, 1999.

18. Chung CT, Niemela SL and Miller RH: One-step preparation of competent Escherichia coli: trasformation and storage of bacterial cells in the same solution. Proc Natl Acad Sci USA 86: 2172-2175, 1989.

19. Sardana G, Dowell B and Diamandis EP: Emerging biomarkers for the diagnosis and prognosis of prostate cancer. Clin Chem 54: 1951-1960, 2008.

20. Bensalah K, Lotan Y, Karam JA, et al: New circulating biomarkers for prostate cancer. Prostate Cancer Prostatic Dis 11: 112-120, 2008.

21. Du H, Masuko-Hongo K, Nakamura H, et al: The prevalence of autoantibodies against cartilage intermediate layer protein, YKL-39, osteopontin, and cyclic citrullinated peptide in patients with early-stage knee osteoarthritis: evidence of a variety of autoimmune processes. Rheumatol Int 26: 35-41, 2005.

22. Watanabe M, Uchida K, Nakagaki K, et al: Anti-cytokine autoantibodies are ubiquitous in healthy individuals. FEBS Lett 581: 2017-2021, 2007.

23. Tomer Y, Sherer Y and Shoenfeld Y: Autoantibodies, autoimmunity and cancer. Oncol Rep 5: 753-761, 1998.

24. Adler AJ: Mechanisms of $\mathrm{T}$ cell tolerance and suppression in cancer mediated by tumor-associated antigens and hormones. Curr Cancer Drug Targets 7: 3-14, 2007.

25. Casiano CA, Mediavilla-Varela $M$ and Tan EM: Tumorassociated antigen arrays for the serological diagnosis of cancer. Mol Cell Proteomics 5: 1745-1759, 2006.

26. Thalmann GN, Sikes RA, Devoll RE, et al: Osteopontin: possible role in prostate cancer progression. Cancer Res 5: 2271-2277, 1999.

27. Bradley SV, Oravecz-Wilson KI, Bougeard G, et al: Serum antibodies to huntingtin interacting protein-1: a new blood test for prostate cancer. Cancer Res 65: 4126-4133, 2005.

28. Pontes ER, Matos LC, da Silva EA, et al: Auto-antibodies in prostate cancer: humoral immune response to antigenic determinants coded by the differentially expressed transcripts FLJ23438 and VAMP3. Prostate 66: 1463-1473, 2006.

29. Shi F, Zhang J, Liu D, et al: Preferential humoral immune response in prostate cancer to cellular proteins p90 and p62 in a panel of tumor-associated antigens. Prostate 63: 252-258, 2005.

30. Sreekumar A, Laxman B, Rhodes DR, et al: Humoral immune response to alpha-methylacyl-CoA racemase and prostate cancer. J Natl Cancer Inst 96: 834-843, 2004.

31. Leman ES and Getzenberg RH: Biomarkers for prostate cancer. J Cell Biochem 108: 3-9, 2009. 